\title{
Risk of Hospitalization for Cancer, Musculoskeletal Disorders, Injuries, or Poisonings Surrounding Widowhood
}

\section{Einiö, Elina}

2019-01

Einiö , E \& Martikainen , P 2019 , ' Risk of Hospitalization for Cancer, Musculoskeletal Disorders, Injuries, or Poisonings Surrounding Widowhood' , American Journal of Epidemiology , vol. 188 , no. 1 , pp. 110-118 . https://doi.org/10.1093/aje/kwy184

http://hdl.handle.net/10138/326161

https://doi.org/10.1093/aje/kwy184

unspecified

acceptedVersion

Downloaded from Helda, University of Helsinki institutional repository.

This is an electronic reprint of the original article.

This reprint may differ from the original in pagination and typographic detail.

Please cite the original version. 


\section{Risk of hospitalization for cancer, musculoskeletal disorders, injuries, or poisonings surrounding widowhood}

Elina Einiö (1, 2, 3) and Pekka Martikainen (1, 3, 4)

1. Population Research Unit, Department of Social Research, University of Helsinki, PO Box 18, FIN-00014 Helsinki, Finland

2. London School of Economics and Political Science, Department of Social Policy, London WC2A 2AE, United Kingdom

3. Max Planck Institute for Demographic Research, Konrad-Zuse-Straße 1, 18057 Rostock, Germany

4. Centre for Health Equity Studies (CHESS), Stockholm University/Karolinska Institutet, Sveavägen 160, 11347 Stockholm, Sweden

E-mails and telephone numbers:

Elina Einiö, DSocSc, elina.einio@helsinki.fi, telephone: +358 50 3199368, fax: +358 919124832

Prof. Pekka Martikainen, PhD, pekka.martikainen@helsinki.fi, telephone: +358 442861946 


\begin{abstract}
Psychological distress has been indicated to affect the risk of death from cardiovascular disease, cancer, and external causes. Mortality from these major causes of death is also known to be elevated after widowhood when distress is at a heightened level. However, surprisingly little is known about changes in health other than mental and cardiac health shortly before widowhood. We used longitudinal data of widowing $(n=19,185)$ and continuously married individuals $(n=105,939)$ to assess the risk of hospitalization for cancer and the external and musculoskeletal causes surrounding widowhood or random dates. The study employed population-averaged logit models for longitudinal data of Finnish older adults aged 65 and over. The results show that hospitalization for injuries had already increased prior to widowhood and clearly peaked after it. The increases were largely related to hospital-treated falls. A similar increasing pattern of findings was not found around a random date for a group of continuously married individuals. Hospitalizations for cancer and musculoskeletal disorders appeared to be unrelated to the process of widowhood. Hospitalizations for poisonings increased after widowhood. The results imply that the process of widowhood is multifaceted and that various types of health changes should be studied separately and already before the actual loss.
\end{abstract}

Abbreviations: SD, standard deviation, ICD-10, International Statistical Classification of Diseases and Related Health Problems, 10th revision 


\section{INTRODUCTION}

The level of psychological distress has been shown to affect the risk of death from cardiovascular disease, cancer, and external causes (1). Mortality from these major causes of death is also known to be elevated after widowhood (2-4), when psychological distress is at a heightened level. In addition to causing psychological distress, it has been suggested that widowhood also leads to an overall crisis of habitualized actions, as the spouse is no longer there to participate in everyday life (5). Accordingly, empirical research has indicated that recently widowed individuals have an elevated risk of institutionalization compared to their married counterparts, and the elevated risk is similar regardless of individuals' education or income (6).

Less is known about changes in health shortly prior to widowhood, although the few longitudinal studies on mental health indicate that depressive symptoms are already on the rise before spousal death $(7,8)$. It is commonly believed that this reflects the multifaceted process of widowhood, with features of caregiving burden and anticipatory grief occurring before the actual loss (9). However, surprisingly little is known about changes in physical health shortly prior to widowhood, although symptoms of different illnesses may react differently to the impending death of a spouse. For instance, illnesses that are sensitive to stress-related changes in the immune system (e.g., cardiovascular disease and cancer) may have already started to aggravate before the actual loss, while the risk of injuries and self-harm may not be elevated until after widowhood when social control maintained by the spouse no longer limits risky health behavior. To our knowledge, however, the only study of changes in physical health shortly prior to widowhood focused on the risk of hospitalization for ischemic heart disease. This study showed that male hospitalizations for cardiac problems are on the rise three months before widowhood (10), but evidence of other medical conditions is meager.

Using unique microdata of older individuals, the present study extends previous work with analyses of the risk of hospitalization for cancer and the external and musculoskeletal causes for the transition to widowhood. The study uses a longitudinal dataset of 24 months before and 24 months after the date of widowhood among those whose spouse died and a corresponding follow- 
up period surrounding a random date for a group of continuously married individuals. The study employs population-averaged logistic models for longitudinal data.

The study examines the hypothesis that the risk of hospitalization for cancer increases shortly prior to widowhood, while that of external causes does not increase until after widowhood. Furthermore, the hypothesis of our study postulates that the risk of hospitalization for musculoskeletal causes is mostly unrelated to widowhood because neither the immunological nor the social control pathways appear to be pertinent.

\section{METHODS}

\section{Data}

The present study was based on a $40 \%$ simple random sample of residents aged 65 or above in Finland collected on December 31, 1997. The longitudinal data, including sociodemographic characteristics and a continuous follow-up of widowhood and mortality from 1998 to 2002, were obtained from Statistics Finland, where these were linked with hospital data from 1996 to 2002 originating from the National Research and Development Centre for Welfare and Health, and with the medicine reimbursement data of the Social Insurance Institution. Statistics Finland utilized personal identification codes to carry out the data linkage (permission numbers: TK-53-1236-15, TK-53-576-04, TK-53-499-05).

We restricted the sample to those 19,185 widowing individuals who resided with their spouse on December 31, 1997 and who experienced the death of a spouse between January 1, 1998 and December 31, 2002 (to-be-widows $n=19,185$ ). The study also used a comparison group of continuously married individuals $(n=105,939)$ who did not experience widowhood during the 5year follow-up.

\section{Hospitalization for cancer, musculoskeletal, and external causes}


The study utilized the national registry of inpatient hospital care from 1996 to 2002 in order to allow an analysis of health changes surrounding widowhood. The hospital data include information on admission dates and corresponding primary diagnoses. The diagnoses data were based on the International Statistical Classification of Diseases and Related Health Problems, 10th revision (11). The study utilized diagnoses data on hospital admissions for malignant cancers (ICD10 codes $\mathrm{C00}-\mathrm{C} 97$ ), disorders of the musculoskeletal system (M00-M99), and injuries and poisonings (S00-S99, T00-T99), except for complications caused by medical or surgical procedures (T80-T88). The selected diagnoses were chosen in order to provide us with novel information on health changes prior to widowhood.

For an additional analysis, we categorized hospitalizations for injuries based on the intent and cause of injury as follows: falls (W00-W19), traffic injuries (V01-V99), poisonings without known self-harm intent (T36-T65 excluding X60-X84, Y870), and injuries and poisonings involving intentional self-harm (X60-X84, Y870). It must be noted, however, that accidental poisonings cannot always be distinguished from self-poisonings (12). In our sample, poisonings were mainly related to the use of medicinal drugs. The prevalences of hospitalization for various causes are displayed in Table 1.

\section{Statistical methods}

Having a continuous timescale for hospitalizations, we were able to construct a population-based medical microdata set surrounding widowhood dates. Widowing individuals were followed for 24 months before and 24 months after widowhood. The time before and after widowhood was divided into 2-month (61-day) periods surrounding a spouse's death. The outcomes were coded as 1 if an individual had hospital admissions attributable to cancer, musculoskeletal, or external causes within a 2-month period, and they were coded as 0 if deemed otherwise. The follow-up was censored at the beginning of the following 2-month period if individuals died or when their follow-up ended on December 31, 2002. 
In order to assess whether hospital use trajectories of widowing individuals followed a specific pattern, a reference population was utilized. This consisted of married individuals who did not experience any marital status transitions from 1998 to 2002. A similar type of reference population has previously been used to study medication trajectories around divorce (13). In our study, continuously married individuals were assigned a random date between January 1, 1998 and December 31, 2002 in order to have a correspondingly centered dataset as that of widowing individuals. If a continuously married person died before a random date, he or she was excluded from the analysis ( $9.3 \%$ of the continuously married). The decision was based on the principle of comparability and clarity around the landmark dates (widowhood vs. random dates), since, by definition, widows-to-be could not die before their spouses.

The study employed population-averaged logit models for longitudinal data. The results are displayed as the adjusted prevalences of hospitalization at each 2-month period surrounding widowhood or a random date for the continuously married. The $95 \%$ confidence intervals are calculated based on the robust standard errors for repeatedly measured outcomes.

Due to the small numbers, injuries involving intentional self-harm and hospital-treated poisonings could not be analyzed using the original division of time into 242 -month intervals. We therefore divided the 48-month follow-up time surrounding widowhood into 8 6-month (183-day) periods to analyze hospitalizations for injuries involving intentional self-harm and poisonings.

In further analyses, we stratified the examination of hospitalization for cancer, musculoskeletal disorders and external causes by sex. These results are displayed in Appendices $1-3$.

\section{Control variables}

The prevalence of hospitalization obtained from population-averaged logit models was adjusted for time-varying covariates for age and period and for time-invariant covariates for sex, educational attainment, region of residence, urbanicity, and three indicators of baseline health. All time-invariant covariates were measured at baseline on December 31, 1997 and introduced into 
the statistical models as categorical variables. Period and age were utilized as categorical variables with 1-year increments in the main analyses (Figures 1-3). Age was used as a continuous variable in other analyses due to data limitations.

Educational attainment was categorized into three groups: tertiary education, intermediate education, and basic education or less. Region of residence was categorized into four regions: Eastern, Southern, Western, and Northern Finland. Helsinki is located in Southern Finland. Urbanicity measured the degree to which a municipality of residence was urban. It was based on a classification provided by Statistics Finland and categorized into three groups: urban, semi-urban, and rural. Urbanicity and region of residence were adjusted for to control for differences in the access to medical care.

We used three dichotomous indicators of chronic conditions, including diabetes, chronic ischemic heart disease, and chronic arrhythmia to assess baseline physical health. Individuals were categorized as having the condition if they were granted the right to receive reimbursement for drug costs due to the condition by December 1997 (14). The Finnish medicine reimbursement system covers all residents, regardless of their income and age (15).

$<$ Table 1>

\section{RESULTS}

Table 1 displays the sociodemographic and health characteristics of continuously married and widowing individuals. Widowing individuals were more likely to be older, female, less educated, and have diabetes than the continuously married. The groups were similar with regard to area of residence and baseline indicators of ischemic heart disease and chronic arrhythmia.

The adjusted prevalence of hospitalization for cancer showed an increasing trend with the follow-up, regardless of whether individuals were widowing or stayed continuously married (Figure 1). The two-month prevalence of hospitalization for cancer increased steadily from $0.4 \%$ 22-24 months before widowhood to $1.0 \% 22-24$ months after widowhood. The corresponding prevalence among the continuously married was $0.4 \%$ and $1.1 \%$. There was no clear finding to 
suggest that widowhood leads to an acceleration of the risk of hospitalization for cancer before or after the death of a spouse.

The risk of hospitalization for musculoskeletal disorders appeared to be unrelated to the process of losing a spouse (Figure 2). The two-month prevalence of hospitalization for musculoskeletal disorders stayed approximately between $0.6 \%$ and $0.7 \%$ throughout the follow-up period among continuously married individuals and between $0.6 \%$ and $0.8 \%$ among widowing individuals.

The prevalence of hospitalization for external causes, including injuries and poisonings, was similar among widowing and continuously married individuals at the study entry: approximately between $0.3-0.4 \%$ during $22-16$ months before widowhood or a random date (Figure 3). However, among widowing individuals, the prevalence rose gradually to $0.5 \% 0-2$ months before widowhood and first peaked at $0.6 \%$ between $0-4$ months after a spouse's death. No similar increasing pattern was observed among the continuously married, whose prevalence of hospitalization for external causes stayed around $0.3-0.4 \%$ throughout the follow-up.

The additional analyses revealed that the increases in the risk of hospitalization for external causes observed before the death of a spouse were largely related to falls (Figure 4). The vast majority of all hospitalizations attributable to external causes were related to falls in the study population (Table 1). The two-month prevalence of hospitalization for injuries stemming from falls increased from $0.2-0.3 \%$ between 18 and 22 months before widowhood to approximately $0.4 \%$ between 0 and 2 months before widowhood. The prevalence clearly peaked $0-2$ months after a spouse's death. A similar increasing pattern was not observed among the continuously married. Further analyses of hospitalizations for external causes indicated that traffic injuries were unrelated to the process of widowhood (Figure 5).

There was no overwhelming evidence to suggest that the process of widowhood leads to the acceleration of the risk of hospitalization for injuries resulting from intentional self-harm (Figure 6). Widowing individuals appeared to have a higher risk of hospitalization for injuries and poisonings involving intentional self-harm compared with the continuously married, but the difference was not significant at any point in time. However, the risk of hospitalization for 
poisonings without a known self-harm intent was higher among recently widowed individuals 0-6 months after the death of a spouse compared with the continuously married $(P$-value $<0.05)$, with whom the risk was the same at the study entry time when both groups were still married (Figure 7).

$<$ Figure 1>

$<$ Figure 2>

$<$ Figure 3>

$<$ Figure 4>

$<$ Figure 5>

$<$ Figure 6>

$<$ Figure 7>

The study findings for cancer, musculoskeletal disorders and external causes were relatively similar for both men and women (Appendices 1-3). However, male hospitalization for external causes showed a marked increase 0-2 months before a spouse's death, whereas female hospitalizations indicated a more modest increase before widowhood. Hospitalizations for external causes showed a marked increase shortly after widowhood for both genders (Appendix 3).

\section{DISCUSSION}

Evidence for the increased risk of acute cardiovascular events and the risk of death after partner bereavement is well established $(16,17)$. In addition, a recent study of widowing individuals showed that men's risk of hospitalization for ischemic heart disease is already on the rise shortly prior to widowhood (10). However, less is known about changes in other aspects of physical health prior to widowhood, although symptoms of different illnesses may react differently to the impending death of a spouse. The present study aimed at bridging this gap in knowledge.

The hypothesis of our study postulated that the risk of accidents and injuries is not elevated until after widowhood. It was based on the idea that individuals would avoid risky health 
behaviors during the sensitive period of a spouse's impending death. However, the main finding of our study somewhat contrasts this hypothesis. The risk of hospitalization for external causes is already on the rise shortly before the death of a spouse. The finding adds to the existing body of knowledge by indicating that widows' elevated mortality stemming from external causes observed after widowhood $(2-4,18)$ not only is due to the experience of widowhood as an unexpected shock but also may be related to the widowhood process that begins prior to a spouse's death. The result is in line with previous studies on mental health acknowledging that depressive symptoms are on the rise prior to widowhood (7). A possible mechanism for this association is that the risk of injuries and accidents rises due to increases in depressive symptoms. Depressive symptoms have been suggested to increase risk-taking behavior and slow reaction times (19). This argument of slow reaction times coincides with our analyses indicating that falls are the primary cause of increases in hospitalizations for external causes observed shortly before widowhood. It is possible that accidental falls are more likely when an older individual is distressed by the impending death of a spouse. Our findings showed no increases in traffic injuries surrounding widowhood, although these could also relate to the consequences of psychological distress. It is thus also possible that the risk of falls occurring prior to widowhood relates to the physical burden of caregiving.

There are several other possible explanations for the increased risk of hospitalization for falls. First, widowing individuals may experience sleep deprivation and disruptions related to the fear of losing a spouse. Sleep disruptions have been shown to cause adverse short-term consequences, including cognitive and performance deficits (20), which could affect the risk of falls (21). Second, widowing individuals may neglect their own physical needs, including nutritional and medication needs, or increase alcohol use, all of which may affect falls. Third, it is also possible that the increased risk of falls surrounding widowhood is related to side effects of psychotropic medications. In a review of medication-related falls in the elderly, Huang et al (21) indicated that the use of psychotropic medication, particularly benzodiazepines and antidepressants, were associated with falls. These drugs may be increasingly prescribed to widowing individuals. Although our study could not directly test side effects of benzodiazepines and antidepressants, our 
data showed that widowing individuals were more likely to have bought these medications than the continuously married. For example, $29 \%$ of widowing women had bought antidepressants (N06A) and 35\% benzodiazepines (N05BA, N05CD, N03AE01) between 1997 and 2002 as compared to $18 \%$ and $26 \%$ of continuously married women. The corresponding figures were $23 \%$ and $29 \%$ for widowing men, and $13 \%$ and $21 \%$ for continuously married men.

Our findings also suggest that the risk of hospitalization for poisonings, excluding the few cases registered as self-poisonings, is elevated 0-6 months after the death of a spouse. This finding resembles those obtained from previous mortality studies (3) and is in line with the hypothesis of the avoidance of risk-taking behavior before a spouse's death. Our findings did not show significant increases in hospital-treated poisonings prior to widowhood. The hypothesis of our study was that the risk of accidents is not elevated until after widowhood, and this appears to hold for the risk of poisonings. However, it is noteworthy that hospitalizations for accidental poisonings cannot always be credibly distinguished from self-poisonings (12). In our study, the risk of hospitalization for poisonings and injuries involving intentional self-harm showed no statistically significant increases around widowhood. This could be related to small numbers or to the poor registration of self-harm intent in older adults.

The results for cancer suggest that older individuals with cancer are vulnerable to the progression of the disease and not necessarily to the process of widowhood per se. This interpretation is supported by the fact that changes in the risk of hospitalization for cancer are on the rise, but it is relatively similar among those who are continuously married and those who become widowed. Our result of widowing individuals is somewhat different from those of mortality studies, indicating an elevated risk of cancer mortality among widows when compared to those who are still married, albeit this effect is small $(3,4)$. However, findings on cancer mortality are not consistent across studies, and some analyses have indicated that cancer mortality is unrelated to widowhood. For example, a recent study from Scotland showed that widowed individuals had a similar risk of death from lung cancer than their married counterparts, after socioeconomic characteristics were controlled for (18). 


\section{Strengths and limitations}

The main strengths of the study are the population-based sample and the continuous timescale for widowhood and hospitalization for cancer, musculoskeletal, and external causes. It allowed us to design a longitudinal dataset surrounding widowhood dates among individuals who lost their spouse and around random dates for the continuously married. Furthermore, many previous studies of widowhood compare the risk of death among the bereaved to that of the married (22). It is likely that selection plays some part in explaining the high risk of death among the bereaved. In our study, changes in the risk of hospitalization were measured repeatedly among widowing and continuously married individuals, which provides an opportunity for a novel approach to bereavement studies.

One of the limitations of the study is that the dataset did not have information on the spouse's cause of death, which could have provided indirect information on the awareness and expectedness of the time to widowhood. However, information on the spouse's cause of death does not necessarily reveal whether the surviving spouse is aware of a spouse's impending death. It has been reported that approximately $15 \%$ of widowing women realized that their husbands' incurable cancers were deadly less than 24 hours before their deaths (23). The awareness time may depend on the quality of contact with medical professionals, the quality of the marital relationship, the patient's fluctuation in health, and the surviving spouse's own characteristics, including education and personality.

Another limitation is that only persons with severe symptoms of the disease are usually admitted to inpatient hospital care. This may lead to an underestimation of the overall level of health problems attributable to a disease. However, we had no reason to believe that this underestimation was different among widowing and continuously married individuals.

\section{CONCLUSIONS}


The current study goes beyond earlier work in several ways. Our study appears to be the first to study changes in the risk of hospitalization for cancer and the external and musculoskeletal causes shortly prior to widowhood. Our results suggest that the risks of hospitalization for cancer and musculoskeletal disorders are unrelated to the process of losing a spouse. On the contrary, the risk of hospitalization for external causes is related to widowhood. The risk of hospitalization for injuries clearly increases after the death of a spouse and already appears to be on the rise before the loss. The latter is a novel contribution to the literature because mortality studies do not allow an analysis of repeated measures of health. The increasing pattern of finding observed before the death of a spouse is mainly related to falls. There are several possible explanations for the rising pattern of hospitalization for falls prior to widowhood. First, these may relate to the harmful effects of depressive symptoms and sleep deprivation already occurring before widowhood or to injuries of caregiving. It is also possible that the use of psychotropic medication contributes to the increased risk of falls surrounding widowhood. The findings of the study imply that widows-to-be should be supported in the overall process of losing a spouse by their significant others and medical professionals in order to avoid hospitalization for injuries both before and after a spouse's death.

\section{ACKNOWLEDGEMENTS}

Author affiliations: Population Research Unit, Department of Social Research, University of Helsinki, Finland (Elina Einiö, Pekka Martikainen); Department of Social Policy, London School of Economics and Political Science, United Kingdom (Elina Einiö); Max Planck Institute for Demographic Research, Rostock, Germany (Elina Einiö, Pekka Martikainen); Centre for Health Equity Studies (CHESS), Stockholm University/Karolinska Institutet, Stockholm, Sweden (Pekka Martikainen)

This study was supported by the Academy of Finland, Finnish Cultural Foundation and Signe and Ane Gyllenberg Foundation. 
Conflict of interest: none declared. 


\section{REFERENCES}

1. Russ TC, Stamatakis E, Hamer M, et al. Association between psychological distress and mortality: individual participant pooled analysis of 10 prospective cohort studies. BMJ. 2012;345:e4933. (doi: 10.1136/bmj.e4933 [doi]).

2. Elwert F, Christakis NA. The effect of widowhood on mortality by the causes of death of both spouses. Am J Public Health. 2008;98(11):2092-8. (doi: 10.2105/AJPH.2007.114348 [doi]).

3. Martikainen P, Valkonen T. Mortality after the death of a spouse: rates and causes of death in a large Finnish cohort. Am J Public Health. 1996;86(8):1087-93.

4. Brenn T, Ytterstad E. Increased risk of death immediately after losing a spouse: Cause-specific mortality following widowhood in Norway. Prev Med. 2016;89:251-6. (doi:

10.1016/j.ypmed.2016.06.019 [doi]).

5. Gronow A. From Habits to Social Structures. Pragmatism and Contemporary Social Theory. Frankfurt am Main: Peter Lang, 2011.

6. Nihtilä E, Martikainen P. Institutionalization of older adults after the death of a spouse. Am J Public Health. 2008;98(7):1228-34. (doi: 10.2105/AJPH.2007.119271).

7. Siflinger B. The Effect of Widowhood on Mental Health - an Analysis of Anticipation Patterns Surrounding the Death of a Spouse. Health Econ. 2016. (doi: 10.1002/hec.3443 [doi]).

8. Monserud MA, Markides KS. Changes in depressive symptoms during widowhood among older Mexican Americans: the role of financial strain, social support, and church attendance. Aging Ment Health. 2016:1-9. (doi: 10.1080/13607863.2015.1132676 [doi]).

9. Vable AM, Subramanian SV, Rist PM, et al. Does the "widowhood effect" precede spousal bereavement? Results from a nationally representative sample of older adults. Am J Geriatr Psychiatry. 2015;23(3):283-92. (doi: 10.1016/j.jagp.2014.05.004 [doi]).

10. Einiö $E$, Moustgaard $H$, Martikainen $P$, et al. Does the risk of hospitalisation for ischaemic heart disease rise already before widowhood? J Epidemiol Community Health. 2017;71(6):599-605. (doi: 
10.1136/jech-2016-207987 [doi]).

11. STAKES. Tautiluokitus ICD-10. Systemaattinen osa (Finnish version of the International Statistical Classification of Diseases and Related Health Problems, tenth revision. Volume 1.). 2nd ed. Helsinki: STAKES (National Research and Development Centre for Welfare and Health), 1999.

12. Remes $H$, Martikainen $P$. Young adult's own and parental social characteristics predict injury morbidity: a register-based follow-up of 135,000 men and women. BMC Public Health. 2015;15:429,015-1763-9. (doi: 10.1186/s12889-015-1763-9 [doi]).

13. Metsa-Simola N, Martikainen P. Divorce and changes in the prevalence of psychotropic medication use: a register-based longitudinal study among middle-aged Finns. Soc Sci Med. 2013;94:71-80. (doi: 10.1016/j.socscimed.2013.06.027 [doi]).

16. Carey IM, Shah SM, DeWilde S, et al. Increased risk of acute cardiovascular events after partner bereavement: a matched cohort study. JAMA Intern Med. 2014;174(4):598-605. (doi: 10.1001/jamainternmed.2013.14558 [doi]).

14. Social Insurance Institution. Statistical Yearbook of the Social Insurance Institution, Finland, 1995, T1:31. Helsinki: Social Insurance Institution, 1996.

15. National Agency for Medicines and Social Insurance Institution. Finnish Statistics on Medicines 2015. Helsinki: National Agency for Medicines and Social Insurance Institution, 2016.

17. Shor E, Roelfs DJ, Curreli M, et al. Widowhood and mortality: a meta-analysis and metaregression. Demography. 2012;49(2):575-606. (doi: 10.1007/s13524-012-0096-x [doi]).

18. Hart CL, Hole DJ, Lawlor DA, et al. Effect of conjugal bereavement on mortality of the bereaved spouse in participants of the Renfrew/Paisley Study. J Epidemiol Community Health. 2007;61(5):455-60. (doi: 61/5/455 [pii]).

19. White DA, Myerson J, Hale S. How Cognitive is Psychomotor Slowing in Depression? Evidence from a Meta-Analysis. Aging Neuropsychol Cogn. 1997;4(3):166-74.

20. Medic G, Wille M, Hemels ME. Short- and long-term health consequences of sleep disruption. 
Nat Sci Sleep. 2017;9:151-61. (doi: 10.2147/NSS.S134864 [doi]).

21. Huang AR, Mallet L, Rochefort CM, et al. Medication-related falls in the elderly: causative factors and preventive strategies. Drugs Aging. 2012;29(5):359-76. (doi: 10.2165/11599460000000000-00000 [doi]).

22. Stroebe M, Schut H, Stroebe W. Health outcomes of bereavement. Lancet. 2007;370(9603):1960-73. (doi: S0140-6736(07)61816-9 [pii]).

23. Valdimarsdottir U, Helgason AR, Furst CJ, et al. Awareness of husband's impending death from cancer and long-term anxiety in widowhood: a nationwide follow-up. Palliat Med. 2004;18(5):432-43. (doi: 10.1191/0269216304pm891oa [doi]).

24. Sasson I, Umberson DJ. Widowhood and depression: new light on gender differences, selection, and psychological adjustment. J Gerontol B Psychol Sci Soc Sci. 2014;69(1):135-45. (doi: 10.1093/geronb/gbt058 [doi]). 